\title{
Generally applicable window settings of low-keV virtual monoenergetic reconstructions in dual-layer CT-angiography of the head and neck
}

\author{
David Zopfs $^{1 \wedge}$, Simon Lennartz ${ }^{1,2} \wedge$, Nuran Abdullayev ${ }^{1} \wedge$, Thorsten Lichtenstein ${ }^{1}$, Kai Roman Laukamp ${ }^{1 \wedge}$, \\ Robert Peter Reimer $^{1} \wedge$, Christoph Kabbasch ${ }^{1} \wedge$, Jan Borggrefe ${ }^{3} \wedge$, Marc Schlamann ${ }^{1}$, Victor Neuhaus ${ }^{1 \# \wedge}$, \\ Nils Große Hokamp ${ }^{1 \# \wedge}$
}

${ }^{1}$ Department of Diagnostic and Interventional Radiology, Faculty of Medicine and University Hospital Cologne, University Cologne, Cologne, Germany; ${ }^{2}$ Department of Radiology, Massachusetts General Hospital, Boston, MA, USA; ${ }^{3}$ Department of Radiology, Neuroradiology and Nuclear Medicine, Johannes Wesling University Hospital, Ruhr University Bochum, Bochum, Germany

\#These authors contributed equally to this work.

Correspondence to: Dr. med. David Zopfs. Department of Diagnostic and Interventional Radiology, University Hospital of Cologne, Kerpener Straße 62, 50937 Cologne, Germany. Email: david.zopfs@uk-koeln.de.

\begin{abstract}
Background: Increased vessel contrast in low-keV virtual monoenergetic images (VMI) in spectral detector CT angiography of the head and neck requires adaption of window settings. Aim of this study was to define generally applicable window settings of low-keV VMI.

Methods: Two radiologists determined ideal subjective window settings for $\mathrm{VMI}_{40-70 \mathrm{keV}}$ in 54 patients. To obtain generally applicable window settings, center and width values were modeled against the attenuation of the internal carotid artery (HUICA). This modeling was performed with and without respect to keV. Subsequently, image quality of $\mathrm{VMI}_{40-70 \mathrm{keV}}$ was assessed using the model-based determined window settings.

Results: With decreasing keV values, HUICA increased significantly in comparison to conventional images (CI) $(\mathrm{P}<0.05$ for $40-60 \mathrm{keV})$. No significant differences between modelled and individually recorded window settings were found confirming validity of the obtained models (P values: $0.2-1.0)$. However, modelling with respect to $\mathrm{keV}$ was marginally less precise.

Conclusions: Window settings of low-keV VMI can be semi-automatically determined in dependency of the ICA attenuation in spectral detector CTA of the head and neck. The reported models are a promising tool to leverage the improved image quality of these images in clinical routine.
\end{abstract}

Keywords: Computed tomography (CT) angiography; angiography; tomography; X-ray computed; head; neck

Submitted Oct 13, 2020. Accepted for publication Mar 25, 2021.

doi: $10.21037 /$ qims-20-1140

View this article at: http://dx.doi.org/10.21037/qims-20-1140

\footnotetext{
^ ORCID: David Zopfs, 0000-0001-9978-7453; Simon Lennartz, 0000-0002-3254-4809; Nuran Abdullayev, 0000-0003-4522-537X; Thorsten Lichtenstein, 0000-0002-4710-2317; Kai Roman Laukamp, 0000-0002-5600-5914; Robert Peter Reimer, 0000-0002-59007181; Christoph Kabbasch, 0000-0003-3712-2258; Jan Borggrefe, 0000-0003-2908-7560; Marc Schlamann, 0000-0002-9940-1503; Victor Neuhaus, 0000-0003-4687-5956; Nils Große Hokamp, 0000-0001-7928-0487.
} 


\section{Introduction}

Imaging of the cervical and cerebral arteries can be performed using sonography, computed tomography (CT), magnetic resonance imaging (MRI) as well as digital subtraction angiography (DSA) (1). Magnetic resonance angiography (MRA) and computed tomography angiography (CTA) are often performed prior to DSA due to faster acquisition times and a lower required level of expenditure $(1,2)$. Yet, in most guidelines and centers, CTA is suggested to primarily evaluate occluded vessels, atherosclerotic plaques or vascular malformations $(3,4)$.

Dual-Energy CT (DECT) allows reconstruction of virtual monoenergetic images (VMI), which are known to improve image quality and increase diagnostic accuracy at low-keV levels compared to conventional images (CI) (5-9). In contrast to other DECT approaches, dual-layer detector CT, also referred to as spectral-detector CT (SDCT), allows for separate registration of high-energy and low-energy photons fully matched in terms of time and location $(10,11)$. Further, reconstruction of VMI is available retrospectively, as DECT information is acquired with every scan $(10,12)$.

In neuroimaging, low-keV VMI reconstructed from DECT have been found to provide an increased contrastto-noise ratio of soft tissue and vessels $(5,6,13-15)$. Particularly, the iodine-associated attenuation is boosted in low-keV VMI; however, this boost may result in blooming of vessels which hampers diagnostic assessment. Therefore, most studies recommend adjustment of window settings when interpreting VMI (10,16-21). Yet, this required modification of window settings is tedious and time consuming possibly limiting the clinical utilization of these images in routine. Hence, the purpose of this study was to determine generally applicable window settings of different $\mathrm{keV}$ VMI reconstructed from SDCT-angiography of the head and neck.

\section{Methods}

\section{Patient selection}

This study was approved by the institutional review board. Written informed consent was waived due to the retrospective character of the study. All procedures performed in studies involving human participants were in accordance with the 1964 Helsinki Declaration and its later amendments or comparable ethical standards. 54 consecutive patients were identified, who were referred for SDCT-derived CTA of the head and neck between July 2017 and June 2018. Inclusion criteria encompassed the following:

(I) Patient age $\geq 18$ years;

(II) Automated contrast agent injection;

(III) Using a standardized scanning protocol as specified below.

Eight patients were excluded, all due to deviations from the standard scanning protocol. All scans were performed based on clinical indications; no scan was performed solely for the purpose of this study.

\section{Imaging protocols}

All scans were performed using a SDCT (IQon Spectral Detector CT, Philips Healthcare, Best, the Netherlands). Patients were examined in a supine, head-first position. Detailed scan parameters are reported in Table 1.

$80 \mathrm{~mL}$ of contrast agent were administered (Accupaque 350, GE Healthcare, Little Chalfont, UK) followed by a $30 \mathrm{~mL}$ saline chaser at $4.0 \mathrm{~mL} / \mathrm{s}$ using an automated injector. Bolus tracking was used to start image acquisition by placing a region of interest in the descending thoracic aorta and using a trigger threshold of $150 \mathrm{HU}$ and a start delay of 5 seconds.

All images were reconstructed in axial plane in a slice thickness of $1 \mathrm{~mm}$ and an increment of 0.45 using a dedicated spectral image reconstruction algorithm with a constant kernel (Spectral, Filter B, Philips Healthcare, Best, the Netherlands). VMI were reconstructed in the range of 40 $70 \mathrm{keV}$ using a $10-\mathrm{keV}$ increment. We refrained from including higher $\mathrm{keV} \mathrm{VMI}$ as iodine-boost and hence vessel contrast are known to decrease with increasing energy level $(6,22)$.

\section{Objective image analysis}

Previous studies highlighted the importance of the attenuation of the vessel in question to determine optimal window width and center in CTA $(10,18,23)$. In line with these, one radiologist (four years of experience) placed ROI in the internal carotid artery (ICA) on CI and attenuation within the vessel was recorded $\left(\mathrm{HU}_{\mathrm{ICA}}\right)$. ROI were then copied to VMI at 40, 50, 60 and $70 \mathrm{keV}$, to warrant constant size and location. Again, attenuation was recorded. The ROI were placed in the enhanced lumen of the vessels and drawn as large as possible; however, inclusion of other structures such as the vessel wall or atherosclerotic plaques was avoided. 
Table 1 Detailed scan parameters of CT-Angiography

\begin{tabular}{lc}
\hline Parameter & Data \\
\hline Scan parameter & 120 \\
Tube voltage (kVp) & 180 \\
Tube current time product (mAs) & 0.5 \\
Rotation time (seconds) & $64 \times 0.625$ \\
Collimation (mm) & $512 \times 512$ \\
Matrix & \\
Contrast agent & 80 \\
Amount of contrast agent (milliliter) & 150 \\
Trigger threshold in the thoracic aorta (HU) & 5 \\
Delay (seconds) & 1 \\
Reconstruction & 0.45 \\
Slice thickness (mm) & \\
Increment &
\end{tabular}

\section{Subjective determination of optimal window settings}

Two subspeciality-trained radiologists with four and six years of experience in neuroradiological imaging, which were not involved in objective analysis independently determined optimal window settings allowing for optimal image quality for assessment of cervical and cerebral vessels in all patients and reconstructions under standardized reading conditions. Image width $(\mathrm{W})$ and center $(\mathrm{C})$ were adjusted and recorded for every $\mathrm{keV}$ level from $40-70 \mathrm{keV}$ in $10 \mathrm{keV}$ increments.

\section{Linear modeling to determine optimal window settings}

To obtain generalizable models, the recorded $\mathrm{C} / \mathrm{W}$ were modeled in dependency of attenuation measured in the internal carotid artery $\left(\mathrm{HU}_{\mathrm{ICA}}\right)$. This resulted in a total of 432 value pairs for each, center and width (2 readers $x$ $4 \mathrm{keV}$-levels $\times 54$ patients). Adopted from earlier studies $(10,20)$, we used two different modelling strategies. In approach 1 two linear equations (for center and width, respectively) were created by plotting all value pairs against $\mathrm{HU}_{\mathrm{ICA}}$ regardless of the reconstruction level. In approach 2 , the recorded center and width were plotted separately against $\mathrm{HU}_{\text {ICA }}$ for each $\mathrm{keV}$ level separately, resulting in 2 linear equations for all four $\mathrm{keV}$ levels. Taking the corresponding $\mathrm{HU}_{\mathrm{ICA}}$, equations were used to predict generalizable window settings for all reconstructions $\left(C_{1} /\right.$
$W_{1}$ respectively $\left.C_{2} / W_{2}\right)$. These were then compared to individually determined window settings to confirm the validity of the models numerically.

\section{Subjective analysis}

To demonstrate utility of semi-automatically obtained window settings, CI and 40-70 keV VMI of all patients were each rated by two radiologists independently. Here, window settings were adjusted according to approach 2 . Readers were blinded with respect to the $\mathrm{keV}$-level and patient data. All readings were conducted in a random order. Reconstructions were ranked from 1-5 (1 being the most appropriate/best reconstruction) regarding vessel contrast, vessel delineation, diagnostic confidence regarding vessel assessment, subjective image noise and overall image quality.

\section{Statistical analyses}

Interval scaled data are reported as mean and standard deviation, ordinal scaled data as median and range. After rejection of normal distribution by Shapiro-Wilk test, further analysis was performed using Wilcoxon test with Steel-Dwass correction for multiple comparisons. Interreader variability was evaluated using the intraclass correlation coefficient (ICC). ICC interpretation was as follows: excellent agreement (ICC $>0.8$ ), good agreement (ICC $>0.6$ ), moderate agreement (ICC $>0.4$ ), and poor agreement (ICC $\leq 0.4)$. P values $\leq 0.05$ were considered significant. Statistical analysis was performed using JMP Software (v14, SAS Institute, Cary, USA).

\section{Results}

Of all 54 patients, 30 patients were female while 24 were male. Mean age was $66.9 \pm 17.9$ years.

\section{Objective image analysis}

Mean attenuation within the ICA was $377.8 \pm 69.4 \mathrm{HU}$ in CI, and showed a continuous increase in VMI from $40-70 \mathrm{keV}$, leading to significantly higher $\mathrm{HU}$ at $40-60 \mathrm{keV}$ as compared to $\mathrm{CI}(1106.1 \pm 224.9 \mathrm{HU} / 721.0 \pm 143.5 \mathrm{HU} / 494.5 \pm 95.7 \mathrm{HU}$, $\mathrm{P}<0.05$ for each, Figure 1).

\section{Subjective determination of optimal window settings}

In comparison to the reference display settings $170 / 600$ 
$(\mathrm{C} / \mathrm{W})$ for $\mathrm{CI}$ of CTA, window settings were altered in all reconstructions by both radiologists. ICC between both radiologists was good to excellent (width: 0.92 , center: 0.79 ). Widest window center and width were found in $40 \mathrm{keV}$ VMI (Table 2).

\section{Linear modeling to determine optimal window settings}

Approach 1, which modeled center and width against $\mathrm{HU}_{\text {ICA }}$ irrespective of the $\mathrm{keV}$-level, showed excellent correlations between $\mathrm{HU}_{\mathrm{ICA}}$ and center as well as between $\mathrm{HU}_{\mathrm{ICA}}$ and width $\left(\mathrm{R}^{2}=0.81\right.$ and $\left.\mathrm{R}^{2}=0.87\right)$. Approach 2 , which modeled center and width against $\mathrm{HU}_{\mathrm{ICA}}$ with respect to the corresponding reconstruction, showed poor to moderate correlations for center and moderate to good correlations for width $\left(\mathrm{R}^{2}=0.21-0.52\right.$ and $\left.\mathrm{R}^{2}=0.47-0.62\right)$. Linear equations of approach 1 and 2 were used to calculate $\mathrm{C}_{1} / \mathrm{W}_{1}$

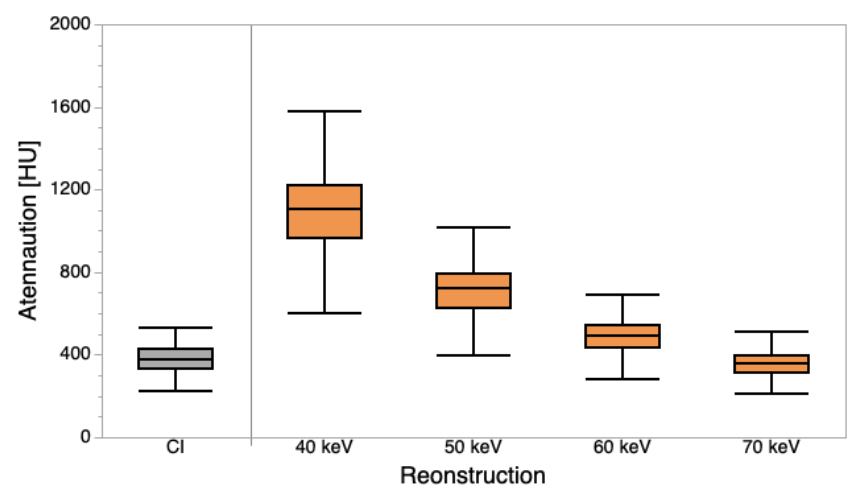

Figure 1 Attenuation in Hounsfield units (HU) within the internal carotid artery in conventional images (CI) and virtual monoenergetic images ranging from $40-70 \mathrm{keV}$. and $\mathrm{C}_{2} / \mathrm{W}_{2}$ respectively.

As compared to the individually determined window settings, no statistically significant differences were found in neither approach 1 nor 2 ( $\mathrm{P}$ values $0.2-1$; Figures 2,3). Figures 2,3 depict low-keV VMI in default tissue window settings and adjusted window settings according to approach 2 .

\section{Subjective analysis}

Low-keV VMI at 40 and $50 \mathrm{keV}$ were more frequently ranked higher in comparison to $60 \mathrm{keV}, 70 \mathrm{keV}$ and CI regarding vessel contrast and vessel delineation (Figure 4). Regarding diagnostic confidence for vessel assessment, $40 \mathrm{keV}$ received most frequent the highest ranking in comparison to all other reconstructions. Equally, both readers ranked $40 \mathrm{keV}$ most frequent as the best reconstruction concerning subjective image noise and overall image quality (Figure 4).

\section{Discussion}

This study investigated model-based, semiautomatic determination of optimal window settings for assessing low-keV VMI reconstructed from SDCT-derived CTA of the head and neck and aimed to provide corresponding reference settings for center and width.

Analogous to previous studies, we found a significantly increasing attenuation within the ICA with decreasing energy levels of VMI $(5,6,24)$. This contrast increase results from the proximity of low-keV VMI to the k-edge of iodine, which is around $33 \mathrm{keV}(5,25)$. Subjective results are in line with the objective findings and confirmed the results of previous studies, which highlighted the potential of an

Table 2 Optimal window settings and equations

\begin{tabular}{|c|c|c|c|c|c|c|c|}
\hline & \multicolumn{3}{|c|}{ Center/Width } & \multicolumn{2}{|r|}{ Equations } & \multicolumn{2}{|c|}{$P$ values (center/width) } \\
\hline $40 \mathrm{keV}$ & $650 / 1509$ & $647 / 1492$ & $650 / 1510$ & Center: & $15.92+0.57 \times \mathrm{HU}_{\mathrm{ICA}} / / 353.42+1.05 \times \mathrm{HU}_{\mathrm{ICA}}$ & $0.985 / 0.847$ & $1,000 / 1.000$ \\
\hline $50 \mathrm{keV}$ & $433 / 1016$ & $434 / 1011$ & $433 / 1016$ & $\begin{array}{c}36.01+0.55 \times \mathrm{HU}_{\text {ICA }} \\
\text { Width: }\end{array}$ & $84.97+0.48 \times \mathrm{HU}_{\mathrm{ICA}} / / 270.12+1.03 \times \mathrm{HU}_{\mathrm{ICA}}$ & $0.988 / 0.969$ & $1,000 / 1.000$ \\
\hline $70 \mathrm{keV}$ & $235 / 538$ & $234 / 558$ & $235 / 538$ & & $92.89+0.40 \times \mathrm{HU}_{\mathrm{ICA}} / / 105.88+1.25 \times \mathrm{HU}_{\mathrm{ICA}}$ & $0.986 / 0.202$ & $1,000 / 1.000$ \\
\hline
\end{tabular}

For every virtual monoenergetic image (VMI) level (40-70 keV) the window settings from both independent readers for the internal carotid artery (ICA) are averaged. Equations for approach 1 and approach 2 and corresponding predicted estimations for center/width are reported. All window settings were compared to the averaged window setting from both readers. No significant differences were found. 

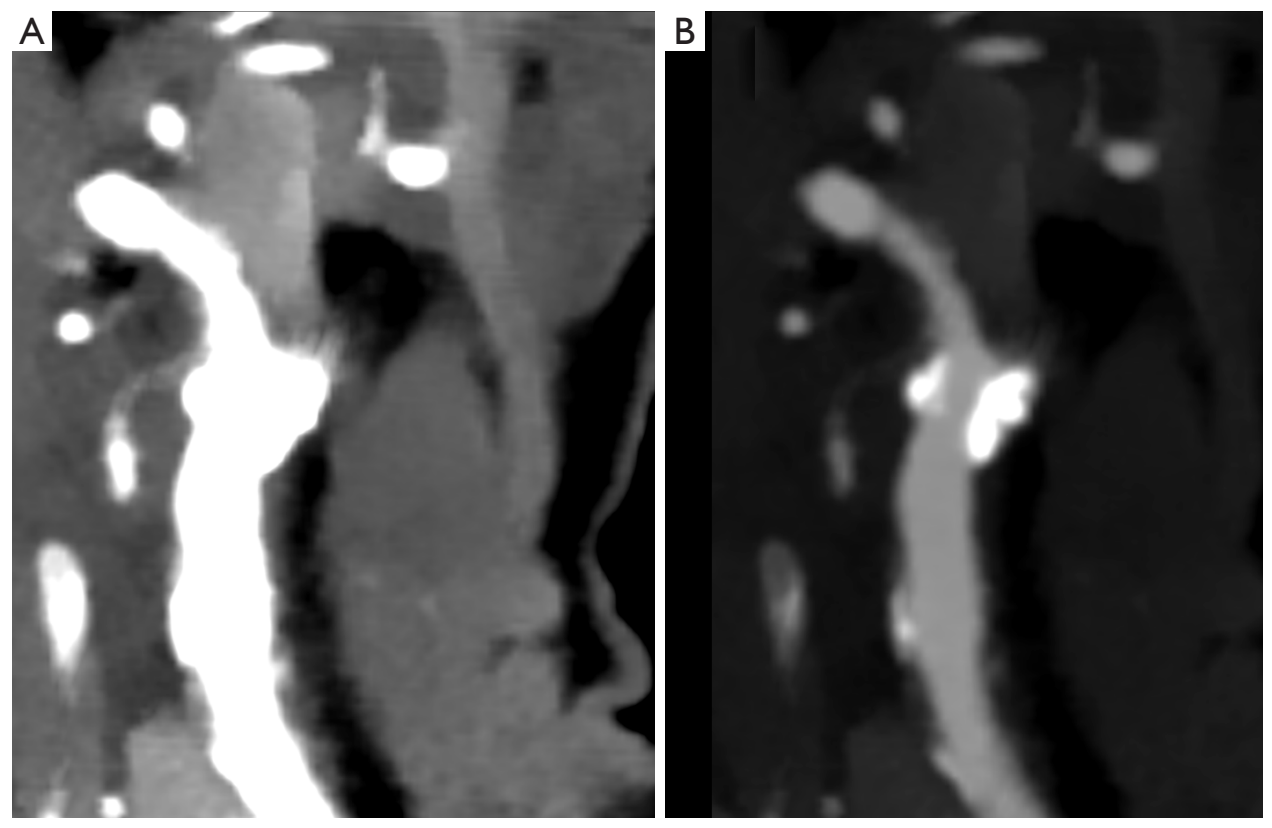

Figure 2 Sagittal view of the common carotid artery and the proximal external carotid artery in $\mathrm{VMI}_{40-\mathrm{keV}}$ with unadjusted window settings as stored within the DICOM-header (A, C: 170, W: 600) and with subjectively adjusted window settings (B, C: 2,437, W: 1,124). VMI, virtual monoenergetic images.

improved vessel assessment of the ICA in contrast enhanced scans at low-keV VMI derived from SDCT, especially in $\mathrm{VMI}$ at $40 \mathrm{keV}(5,6)$. A growing body of evidence indicates that utilization of SDCT and DECT in general is beneficial in daily routine $(5-10,14,18-20,24)$. Different studies underlined the need for adjustment of window settings in order to achieve the best diagnostic image quality in dual energy CT (10,16-18,20,21). D'Angelo et al. investigated optimal window settings for VMI of dual-source DECT derived CTA images of the head and neck and found ideal $\mathrm{C} / \mathrm{W}$ values for $\mathrm{VMI}_{40 \mathrm{keV}}$ within the same range as ours (26), indicating a generalizability of our results. However, the D'Angelo et al. limited their analysis to $\mathrm{VMI}_{40 \mathrm{keV}}$ and $\mathrm{VMI}_{70 \mathrm{ke}}$, while we investigated $\mathrm{VMI}$ at 50 and $60 \mathrm{keV}$, also. To the best of our knowledge, this is the first study to focus on the adjustment of window settings in SDCT-derived CTA examinations of the head and neck.

In contrast to different previous studies, in which optimal window setting were solely assessed based on a visual assessment, we used a reader-based assessment to obtain a linear modelling approach which allows to estimate window settings semi-automatically $(10,21,23)$. By taking the attenuation of the internal carotid artery $\left(\mathrm{HU}_{\mathrm{ICA}}\right)$ into account when calculating the linear models, we aimed to take interindividual differences regarding the contrast of the ICA into account, which may be caused by the cardiovascular output, vessel stenosis and/or the injection rate of the contrast agent.

Default window settings vary between institutions, yet are commonly acknowledged as a width of 600 and a center of 170 for CTA for the head and neck. This information is stored within the DICOM header of each image allowing for automated display. However, due to the pronounced increase in contrast in low-keV VMI and the resulting blooming particularly of iodine, these window settings are not applicable to low-keV imaging and for a diagnostic assessment they need to be adjusted. Accordingly, we found a significant discrepancy between default window settings and both subjectively determined and model-based optimal window settings. Approach 1, which modeled $\mathrm{HU}_{\mathrm{ICA}}$ against all subjectively determined $\mathrm{C} / \mathrm{W}$ values irrespective of the energy level of the reconstruction, thus providing valid estimations for window center and window width irrespective of the $\mathrm{keV}$ level. Approach 2, on the other hand, considered the energy level and provided slightly more accurate results. Differences obtained from approach 1 ranged from 1-20 HU in comparison to the individually determined window setting. Approach 2 allowed for an accurate estimation of optimal window setting with almost no differences in as compared to individually determined 


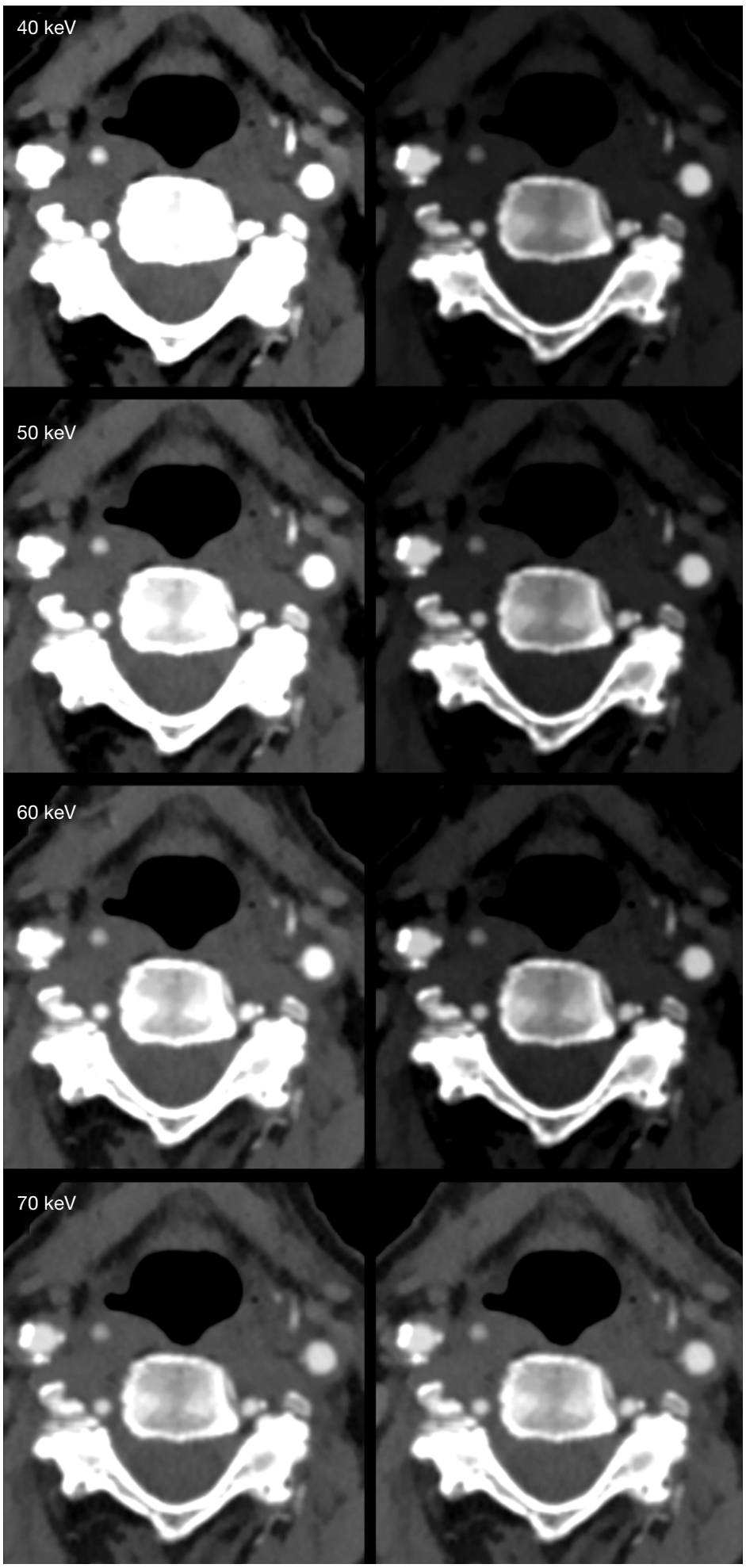

Figure 3 Axial images of the common carotid artery and the vertebral arteries in $\mathrm{VMI}_{40-70 \mathrm{keV}}$ with unadjusted window settings as stored within the DICOM-header (left column) and adjusted window settings according to approach 2 (right column). Window settings in the left column C: 170, W: 600. Window settings in the right column: $40 \mathrm{keV}$, C: 700, W: 1612; $50 \mathrm{keV}, \mathrm{C}: 467, \mathrm{~W}: 1085 ; 60 \mathrm{keV}$, C: 330 , W: 775; $70 \mathrm{keV}, \mathrm{C}: 247, \mathrm{~W}: 587$. 

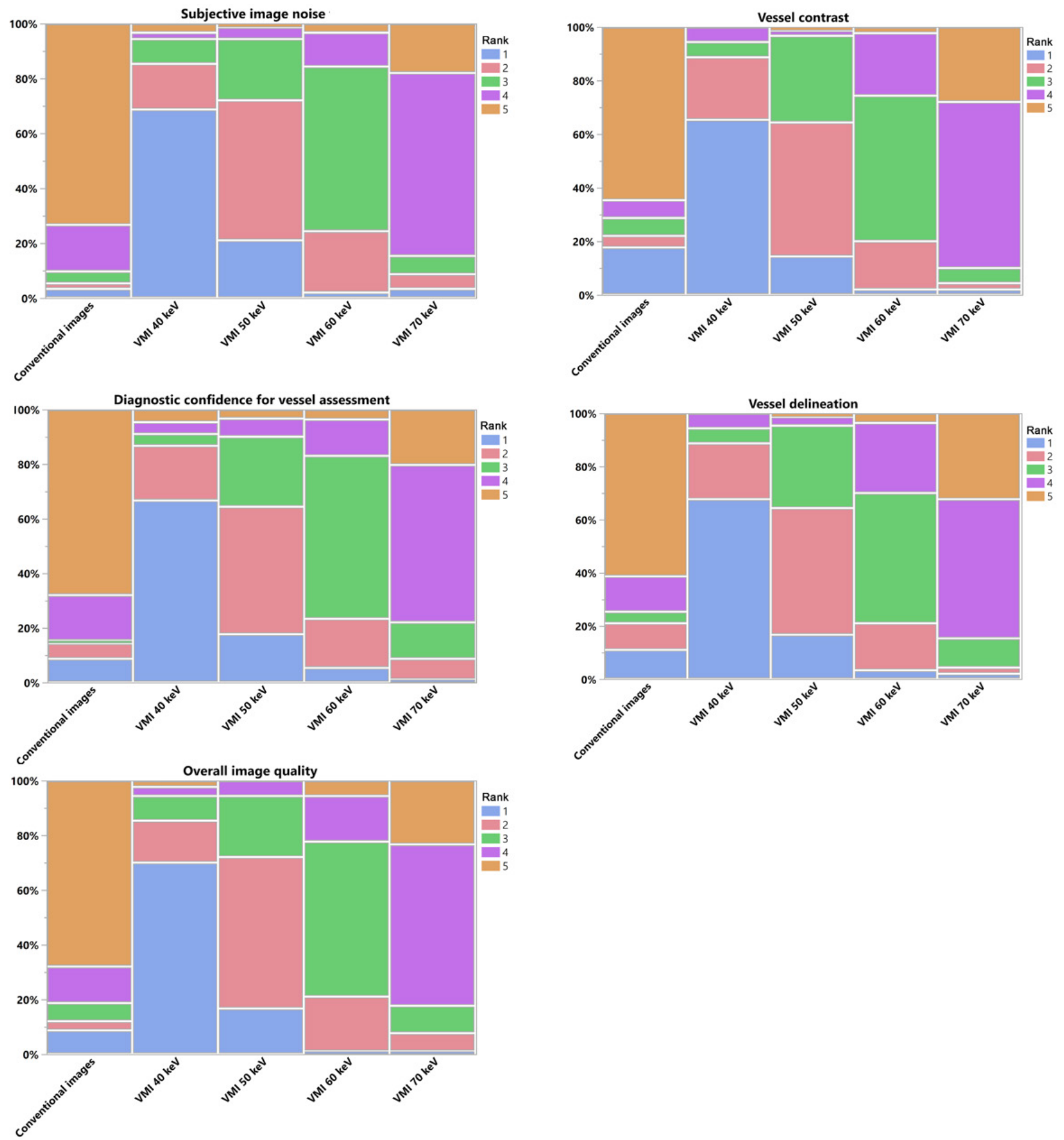

Figure 4 Frequency of subjective rankings regarding overall image quality and subjective image noise, assessment of vessel delineation, vessel contrast as well as diagnostic confidence for vessel assessment in the internal carotid artery at different low-keV virtual monoenergetic images $\left(\mathrm{VMI}_{40-70 \mathrm{keV}}\right)$ and conventional images. 
window settings (range: $1 \mathrm{HU}$ ). After all, either differences were not found to reach significance as compared to individually determined settings. Therefore, a reliable calculation of optimal window settings using the proposed linear model with regard to $\mathrm{HU}_{\text {ICA }}$ seems feasible. Although window center and width can be adjusted in real time in daily radiological routine, a free-handed adjustment of window settings is likely time-consuming and may limit the actual usage of low-keV VMI, whose image quality has been reported to be superior compared to CI reconstructions $(5-7,15)$. Therefore, we suggest embedding the herewith provided window settings into the DICOM header of VMI. For the DECT and scan protocol used in this study, individualized values are suggested in Table 2.

Beside the retrospective study design, this study is limited in patient size. Additionally, our approaches are limited to similar CT-scanners and acquisition protocols. We used a two-reader approach to identify appropriate window settings serving as input for modelling. Both readers showed a good to excellent ICC substantiating the validity of values provided in this study. In numerical validation, no differences between predicted and individually determined window settings was found. Thus, we did not use an additional set of readers to validate the models. Further, we considered $\mathrm{HU}_{\mathrm{ICA}}$ the most important determinant of appropriate window settings. However, in the presence of significant atherosclerotic plaque additional adjustments may be necessary. Further studies should focus on a fully automated configuration of window settings to save time and facilitate an increased use of low$\mathrm{keV}$ VMI in daily clinical routine.

In conclusion, this study defines reference values for window width and window center in different low-keV VMI from SDCT-derived CTA of the head and neck. The determined optimal window settings depend on the attenuation within the ICA. Therefore, an accurate adjustment of the DICOM header defaults for low-keV VMI of SDCTderived CTA examinations of the head and neck should be considered to promote the usage of VMI in clinical routine.

\section{Acknowledgments}

Funding: This work was supported by the Else KrönerFresenius Stiftung (2018_EKMS.34 to Nils Große Hokamp).

\section{Footnote}

Conflicts of Interest: All authors have completed the ICMJE uniform disclosure form (available at http://dx.doi. org/10.21037/qims-20-1140). SL and NGH received research support from Philips Healthcare during the conduct of this study. NGH and JB received speakers' honoraria from Philips Healthcare. NGH reports that this work was supported by the Else Kröner-Fresenius Stiftung (2018_EKMS.34 to NGH). The other authors have no conflicts of interest to declare.

Etbical Statement: The authors are accountable for all aspects of the work in ensuring that questions related to the accuracy or integrity of any part of the work are appropriately investigated and resolved. The study was conducted in accordance with the Declaration of Helsinki (as revised in 2013). This retrospective study was approved by the local institutional review board. Written informed consent was waived due to the retrospective character of the study.

Open Access Statement: This is an Open Access article distributed in accordance with the Creative Commons Attribution-NonCommercial-NoDerivs 4.0 International License (CC BY-NC-ND 4.0), which permits the noncommercial replication and distribution of the article with the strict proviso that no changes or edits are made and the original work is properly cited (including links to both the formal publication through the relevant DOI and the license). See: https://creativecommons.org/licenses/by-nc-nd/4.0/.

\section{References}

1. Salmela MB, Mortazavi S, Jagadeesan BD, Broderick DF, Burns J, Deshmukh TK, Harvey HB, Hoang J, Hunt CH, Kennedy TA, Khalessi AA, Mack W, Patel ND, Perlmutter JS, Policeni B, Schroeder JW, Setzen G, Whitehead MT, Cornelius RS, Corey AS. ACR Appropriateness Criteria ${ }^{\circledR}$ Cerebrovascular Disease. J Am Coll Radiol 2017;14:S34-61.

2. Hankey GJ, Warlow CP, Sellar RJ. Cerebral angiographic risk in mild cerebrovascular disease. Stroke 1990;21:209-22.

3. Lev MH, Farkas J, Rodriguez VR, Schwamm LH, Hunter GJ, Putman CM, Rordorf GA, Buonanno FS, Budzik R, Koroshetz WJ, Gonzalez RG. CT angiography in the rapid triage of patients with hyperacute stroke to intraarterial thrombolysis: accuracy in the detection of large vessel thrombus. J Comput Assist Tomogr 2001;25:520-8.

4. Smith WS, Roberts HC, Chuang NA, Ong KC, Lee TJ, Johnston SC, Dillon WP. Safety and feasibility of a CT protocol for acute stroke: combined CT, CT angiography, 
and CT perfusion imaging in 53 consecutive patients. AJNR Am J Neuroradiol 2003;24:688-90.

5. Zopfs D, Lennartz S, Laukamp K, Große Hokamp N, Mpotsaris A, Maintz D, Borggrefe J, Neuhaus V. Improved depiction of atherosclerotic carotid artery stenosis in virtual monoenergetic reconstructions of venous phase dual-layer computed tomography in comparison to polyenergetic reconstructions. Eur J Radiol 2018;100:36-42.

6. Neuhaus V, Große Hokamp N, Abdullayev N, Maus V, Kabbasch C, Mpotsaris A, Maintz D, Borggrefe J. Comparison of virtual monoenergetic and polyenergetic images reconstructed from dual-layer detector CT angiography of the head and neck. Eur Radiol 2018;28:1102-10.

7. Schneider D, Apfaltrer P, Sudarski S, Nance JW, Haubenreisser H, Fink C, Schoenberg SO, Henzler T. Optimization of kiloelectron volt settings in cerebral and cervical dual-energy CT angiography determined with virtual monoenergetic imaging. Acad Radiol 2014;21:431-6.

8. Zhao L, Li F, Zhang Z, Zhang Z, Jiang Y, Wang X, Gu J, Li D. Assessment of an advanced virtual monoenergetic reconstruction technique in cerebral and cervical angiography with third-generation dual-source CT: Feasibility of using low-concentration contrast medium. Eur Radiol 2018;28:4379-88.

9. Zopfs D, Laukamp KR, Pinto Dos Santos D, Sokolowski M, Große Hokamp N, Maintz D, Borggrefe J, Persigehl T, Lennartz S. Low-keV virtual monoenergetic imaging reconstructions of excretory phase spectral dual-energy CT in patients with urothelial carcinoma: A feasibility study. Eur J Radiol 2019;116:135-43.

10. Große Hokamp N, Kessner R, van Hedent S, Graner FP, Gupta A, Gilkeson R. Spectral Detector Computed Tomography Pulmonary Angiography: Improved Diagnostic Assessment and Automated Estimation of Window Settings Angiography of Pulmonary Arteries From Novel Spectral Detector Computed Tomography Provides Improved Image Quality if Settings are Adjusted. J Comput Assist Tomogr 2018;42:850-7.

11. Maass C, Baer M, Kachelriess M. Image-based dual energy CT using optimized precorrection functions: a practical new approach of material decomposition in image domain. Med Phys 2009;36:3818-29.

12. Rassouli N, Etesami M, Dhanantwari A, Rajiah P. Detector-based spectral CT with a novel dual-layer technology: principles and applications. Insights Imaging 2017;8:589-98.
13. Pomerantz SR, Kamalian S, Zhang D, Gupta R, Rapalino O, Sahani DV, Lev MH. Virtual monochromatic reconstruction of dual-energy unenhanced head CT at $65-75 \mathrm{keV}$ maximizes image quality compared with conventional polychromatic CT. Radiology 2013;266:318-25.

14. Neuhaus V, Abdullayev N, Große Hokamp N, Pahn G, Kabbasch C, Mpotsaris A, Maintz D, Borggrefe J. Improvement of Image Quality in Unenhanced DualLayer CT of the Head Using Virtual Monoenergetic Images Compared With Polyenergetic Single-Energy CT. Invest Radiol 2017;52:470-6.

15. Leithner D, Mahmoudi S, Wichmann JL, Martin SS, Lenga L, Albrecht MH, Booz C, Arendt CT, Beeres M, D'Angelo T, Bodelle B, Vogl TJ, Scholtz J-E. Evaluation of virtual monoenergetic imaging algorithms for dualenergy carotid and intracerebral CT angiography: Effects on image quality, artefacts and diagnostic performance for the detection of stenosis. Eur J Radiol 2018;99:111-7.

16. Hickethier T, Iuga A-I, Lennartz S, Hauger M, Byrtus J, Luetkens JA, Haneder S, Maintz D, Doerner J. Virtual Monoenergetic Images From a Novel Dual-Layer Spectral Detector Computed Tomography Scanner in Portal Venous Phase: Adjusted Window Settings Depending on Assessment Focus Are Essential for Image Interpretation. J Comput Assist Tomogr 2018;42:350-6.

17. D'Angelo T, Bucher AM, Lenga L, Arendt CT, Peterke JL, Caruso D, Mazziotti S, Blandino A, Ascenti G, Othman AE, Martin SS, Leithner D, Vogl TJ, Wichmann JL. Optimisation of window settings for traditional and noise-optimised virtual monoenergetic imaging in dualenergy computed tomography pulmonary angiography. Eur Radiol 2018;28:1393-401.

18. Caruso D, Parinella AH, Schoepf UJ, Stroebel MH, Mangold S, Wichmann JL, Varga-Szemes A, Ball BD, Santis D de, Laghi A, Cecco CN de. Optimization of window settings for standard and advanced virtual monoenergetic imaging in abdominal dual-energy CT angiography. Abdom Radiol (NY) 2017;42:772-80.

19. Bae K, Jeon KN, Cho SB, Park SE, Moon JI, Baek HJ, Choi BH. Improved Opacification of a Suboptimally Enhanced Pulmonary Artery in Chest CT: Experience Using a Dual-Layer Detector Spectral CT. AJR Am J Roentgenol 2018;210:734-41.

20. Große Hokamp N, Obmann VC, Kessner R, Gilkeson RC, Gupta A, Persigehl T, Haneder S, Ramaiya N. Virtual Monoenergetic Images for Diagnostic Assessment of Hypodense Lesions Within the Liver: Semiautomatic 
Estimation of Window Settings Using Linear Models. J

Comput Assist Tomogr 2018;42:925-31.

21. Iuga AI, Doerner J, Siedek F, Haneder S, Byrtus J, Luetkens JA, Maintz D, Hickethier T. Computed tomography pulmonary angiograms using a novel duallayer spectral detector: Adjusted window settings are essential for diagnostic image quality. Medicine (Baltimore) 2019;98:e16606.

22. Große Hokamp N, Höink AJ, Doerner J, Jordan DW, Pahn G, Persigehl T, Maintz D, Haneder S. Assessment of arterially hyper-enhancing liver lesions using virtual monoenergetic images from spectral detector CT: phantom and patient experience. Abdom Radiol (NY) 2018;43:2066-74.

23. De Cecco CN, Caruso D, Schoepf UJ, Wichmann JL, Ter Louw JR, Perry JD, Picard MM, Schaefer AR, Parker LW, Hardie AD. Optimization of window settings for virtual monoenergetic imaging in dual-energy CT of the liver:

Cite this article as: Zopfs D, Lennartz S, Abdullayev N, Lichtenstein T, Laukamp KR, Reimer RP, Kabbasch C, Borggrefe J, Schlamann M, Neuhaus V, Große Hokamp N. Generally applicable window settings of low-keV virtual monoenergetic reconstructions in dual-layer CT-angiography of the head and neck. Quant Imaging Med Surg 2021;11(8):34083417. doi: 10.21037/qims-20-1140
A multi-reader evaluation of standard monoenergetic and advanced imaged-based monoenergetic datasets. Eur J Radiol 2016;85:695-9.

24. Albrecht MH, Vogl TJ, Martin SS, Nance JW, Duguay TM, Wichmann JL, Cecco CN de, Varga-Szemes A, van Assen M, Tesche C, Schoepf UJ. Review of Clinical Applications for Virtual Monoenergetic Dual-Energy CT. Radiology 2019;293:260-71.

25. McCollough CH, Leng S, Yu L, Fletcher JG. Dual- and Multi-Energy CT: Principles, Technical Approaches, and Clinical Applications. Radiology 2015;276:637-53.

26. D'Angelo T, Lenga L, Arendt CT, Bucher AM, Peterke JL, Caruso D, Mazziotti S, Ascenti G, Blandino A, Othman AE, Martin SS, Albrecht MH, Bodelle B, Vogl TJ, Wichmann JL. Carotid and cerebrovascular dualenergy computed tomography angiography: Optimization of window settings for virtual monoenergetic imaging reconstruction. Eur J Radiol 2020;130:109166. 